OPEN ACCESS

Edited by:

Kathrin Eller,

Medical University of Graz, Austria

Reviewed by:

Manfred Hecking,

Medical University of Vienna, Austria

Gaurav Gupta,

Virginia Commonwealth University,

United States

*Correspondence:

Turun Song

332441983@qq.com

Tao Lin

kidney5@163.com

tThese authors have contributed equally to this work and share first

authorship

Specialty section:

This article was submitted to

Nephrology,

a section of the journal

Frontiers in Medicine

Received: 24 October 2021 Accepted: 08 December 2021 Published: 23 December 2021

Citation:

Yin S, Ma M, Huang Z, Fan Y, Wang X, Song $T$ and Lin T (2021) Ameliorating

Metabolic Profiles After Kidney

Transplantation: A Protocol for an

Open-Label, Prospective,

Randomized, 3-Arm, Controlled Trial.

Front. Med. 8:800872.

doi: 10.3389/fmed.2021.800872

\section{Ameliorating Metabolic Profiles After Kidney Transplantation: A Protocol for an Open-Label, Prospective, Randomized, 3-Arm, Controlled Trial}

\author{
Saifu Yin ${ }^{1,2,3+}$, Ming Ma ${ }^{1,2,3+}$, Zhongli Huang ${ }^{1,2,3}$, Yu Fan ${ }^{1,2,3}$, Xianding Wang ${ }^{1,2,3}$, \\ Turun Song ${ }^{1,2,3 *}$ and Tao Lin ${ }^{1,2,3 *}$ \\ ${ }^{1}$ Department of Urology, West China Hospital, Sichuan University, Chengdu, China, ${ }^{2}$ Institute of Urology, West China \\ Hospital, Sichuan University, Chengdu, China, ${ }^{3}$ Organ Transplant Center, West China Hospital, Sichuan University, Chengdu, \\ China
}

Aim: High prevalence of metabolic disorders causes higher risk of cardiovascular diseases after kidney transplantation (KT), which remains the main burden impairing short-term and long-term survival. This open-label, prospective, randomized, 3-arm, controlled trial will evaluate the safety, tolerability and efficacy of metformin and empagliflozin in ameliorating metabolic profiles after $\mathrm{KT}$.

Methods: After a screening assessment, eligible patients with an estimated glomerular filtration rate (eGFR) $>45 \mathrm{~mL} / \mathrm{min} / 1.73 \mathrm{~m} 2$ are randomly assigned to standard triple immunosuppression alone, standard immunosuppression plus metformin (500 mg twice daily), standard immunosuppression plus empagliflozin ( $25 \mathrm{mg}$ once daily) from discharge. The primary endpoint is the differences in the visceral-to-subcutaneous fat area ratio over 12 months, evaluated by magnetic resonance imaging (MRI). Secondary outcomes include kidney graft function, glycometabolism, lipid metabolism, and inflammatory parameters. The trial will enroll 105 kidney transplant recipients, providing $90 \%$ power to detect the difference at $5 \%$ significance.

Keywords: metformin, empagliflozin, kidney transplantation, metabolic disorders, clinical trial

\section{INTRODUCTION}

Advances in patient selection, organ procurement and preservation, surgical technique, immunosuppression, and infection prevention have conferred significant improvement in rejection, infection, and subsequently decreased cause-specific graft failure rates after kidney transplantation (KT) (1). However, cardiovascular diseases (CVD) remain the main burden impairing both short-and long-term patient survival (2). Compared with the general population, conventional CVD risk factors, including obesity, liver and muscle insulin resistance, dyslipidemia, hypertension, and diabetes mellitus, are all highly prevalent in this population for long-term exposure to steroids and calcineurin inhibitors (3-6).

Previous studies demonstrated that adenosine $5^{\prime}$-monophosphate (AMP)-activated protein kinase (AMPK) is a central regulator of multiple metabolic pathways and a key player in regulating cellular energy metabolism $(7,8)$. Activation of AMPK by pharmacological agents may hold a considerable potential to reverse the metabolic abnormalities in chronic 
metabolic diseases $(9,10)$. Metformin, a widely used antidiabetic drug, acts as an AMPK activator by inhibiting complex I of the mitochondrial electron transport chain in many tissues, including adipose, skeletal muscle, and heart (11). A recent small clinical trial observed that metformin administration can safely ameliorate metabolic profiles in glucocorticoid-treated patients with inflammatory disease but without pre-existing diabetes (12). In addition, another antidiabetic drug sodium-glucosecotransporter-2 (SGLT-2) inhibitors can improve metabolic parameters and cardiovascular risk in patients with or without diabetes in pre-clinical and clinical studies (13, 14, 14, 15). Another small clinical trial even reported that compared to metformin, significant improvement in anthropometric parameters and body composition, in overweight and obese women with polycystic ovary syndrome after treatment with empagliflozin (16). Hence, metformin and SGLT2 inhibitors may be used as potential adjuvant therapies to improve metabolic disorders after KT.

Although several preliminary clinical trials showed that metformin and SGLT- 2 inhibitors can be used safely and improve glucose control after KT, but they are small-sample sized and only include patients with diabetes (17-19). We will conduct a prospective clinical trial with the aim of exploring their roles in improving metabolic profiles.

\section{METHODS AND ANALYSES}

\section{Study Design}

This study is designed as a 12-month, single-center, prospective, 3-arm, open-label, randomized clinical trial (Figure 1). This study has been registered at www.clinicaltrials.gov (NCT05013112) and is conducted in accordance with the Declaration of Helsinki and local regulations. Selected patients are recruited if they meet the following inclusion criteria: (1) living-donor kidney transplantation; (2) eGFR level $>45$ $\mathrm{ml} / \mathrm{min} / 1.73 \mathrm{~m} 2$ at discharge; (3) $18<$ Age $<65$ years; (4) receiving standard triad immunosuppressive regimen. Our key exclusion criteria are as follows: (1) previous therapy with metformin or SGLT 2 over the previous 3 months; (2) pre-transplant diabetes; (3) alanine aminotransferase (ALT) or aspartate aminotransferase (AST) $>2.5$ or more of upper limit of normal; (4) Combined with $\mathrm{HBV} / \mathrm{HCV} / \mathrm{HIV}$ infection in the donor or recipient; (5) Malignancy history in the donor and recipient; (6) organ transplant history in the recipient. In addition, patients will exit from this clinical trial: (1) Serious deviation from clinical trial protocol; (2) Due to serious adverse reactions, the drug must be stopped in advance; (3) Withdrawal of informed consent; (4) Poor compliance and failure to use drugs as planned; (5) Lost to follow up.

\section{Randomization and Masking}

Eligible participants are randomly allocated to standard immunosuppression (placebo group), standard immunosuppression plus metformin (metformin group), or standard immunosuppression plus Empagliflozin (Empagliflozin group). The randomization sequence is created using a random number generation function and allocation to each group is done through block randomization in a 1:1:1 ratio. The budesonide is open label.

\section{Procedures}

Metformin is administered $500 \mathrm{mg}$ twice daily orally from discharge based on the previous preliminary clinical trial (17). Empagliflozin is administered $25 \mathrm{mg}$ once daily orally from discharge $(18,20)$. This will commence at randomization and continue for 12 months. The decision to continue with metformin beyond 12 months of follow-up is left to the discretion of the caring physician. In addition, during the follow-up, when kidney transplant recipients are diagnosed with PTDM, insulin therapy were adopted to reduce the glucose level at 6.1-6.7 mmol/L based on Chinese Management Guideline of post-transplantation diabetes mellitus after kidney transplantation (21).

Immunosuppressive regimen has been reported in our previous study (22). In brief, all participants will receive standardized triad immunosuppression, consisting of tacrolimus, mycophenolate mofetil/enteric-coated mycophenolate sodium, and steroids. Tacrolimus trough levels are measured by the enzyme multiplied immunoassay technique before breakfast and dose administration in the morning. Recommended tacrolimus trough levels are $5-10 \mathrm{ng} / \mathrm{ml}$. MMF is started at $1000 \mathrm{mg}$ one night before operation and maintained at $1000 \mathrm{mg}$ twice daily with area under curve of mycophenolate mofetil maintained at $30-70 \mathrm{mg} / \mathrm{h} \cdot \mathrm{L}-1$. If EC-MPS is selected, $720 \mathrm{mg}$ is given the night before surgery and $720 \mathrm{mg}$ bid thereafter. Methylprednisolone is injected intravenously at $500 \mathrm{mg}$ during the operation and $200 \mathrm{mg}$ per day 3 days after the operation. Then $60 \mathrm{mg}$ prednisone is initiated, and gradually taper to $5-10 \mathrm{mg}$ per day for maintenance.

\section{Data Collection and Clinical Outcomes}

Standard demographic, clinical and laboratory data (including medication details) will be collected prospectively from electronic medical records. Recipients are advised to receive routine followup weekly in months $0-3$, every 2 weeks in month 4-6, monthly within 6-12 months, and every 3 months thereafter.

The general aim of this study is to determine the safety of metformin and empagliflozin in kidney transplant recipients and explore the role of metformin and empagliflozin treatment in improving metabolic profiles. Based on previous study, visceralto-subcutaneous fat area ratio, evaluated by magnetic resonance imaging (MRI), is generally reported as a surrogate for metabolic risk and is markedly raised in patients with long-term exposure to steroids $(23,24)$. Hence, the primary outcome is the differences in the visceral-to-subcutaneous fat area ratio over 12 months among three groups. The visceral-to-subcutaneous fat area ratio will be measured at transplantation, and post-transplant 3, 6, 9, and 12 months. Kidney graft function will be evaluated when they receive routine follow-up. Secondary outcomes included glycometabolism (fasting plasma glucose, fasting insulin levels, 2-h post-prandial insulin levels, hemoglobin A1c, and insulin beta-cell function [indicated by the homeostasis model assessment of $\beta$ cell function]), lipid metabolism (serum triglyceride, non-high-density lipoprotein, low-density 
lipoprotein, total cholesterol), inflammatory parameters (Creactive protein, interleukin-6, and tumor necrosis factor alpha) (Table 1). We will also examine cardiovascular disease and microvascular complications, including the following: major adverse cardiovascular events; all-cause mortality; atherosclerotic cardiovascular (ASCV) death; hospitalization or death from coronary artery disease, ischemic stroke, or heart failure; observable background diabetic retinopathy; and diabetic peripheral neuropathy.

\section{Sample Size Estimation}

Sample size calculation is based on the primary outcome. According to the previous study, the visceral-to-subcutaneous adipose tissue area ratio was $0.83(\mathrm{SD}=0.48 ; n=58)$ for kidney transplant recipients (25). The ratio is estimated to be half of that in the intervention group. The estimated sample sizes for $90 \%$ power at $5 \%$ significance is 28 patients in each group.
Considering an estimated $20 \%$ dropout rate, we assure that the sample size exceeds the minimal number needed to ensure the validity of the mean, effect size and rationale of feasibility. Therefore, 105 individuals (35 each group) are expected to be enrolled.

\section{Statistical Analysis}

Two populations will be used in the analyses. The intentionto-treat (ITT) population will include all participants who have been randomly assigned, whereas the per-protocol (PP) population will include all participants who accomplish the entire intervention. The baseline characteristics of the study will be summarized as the means \pm standard deviations for parametrically distributed data, geometric mean values (and 95\% confidence intervals) for non-parametrically distributed data, and numbers (percentages) for categorical data.

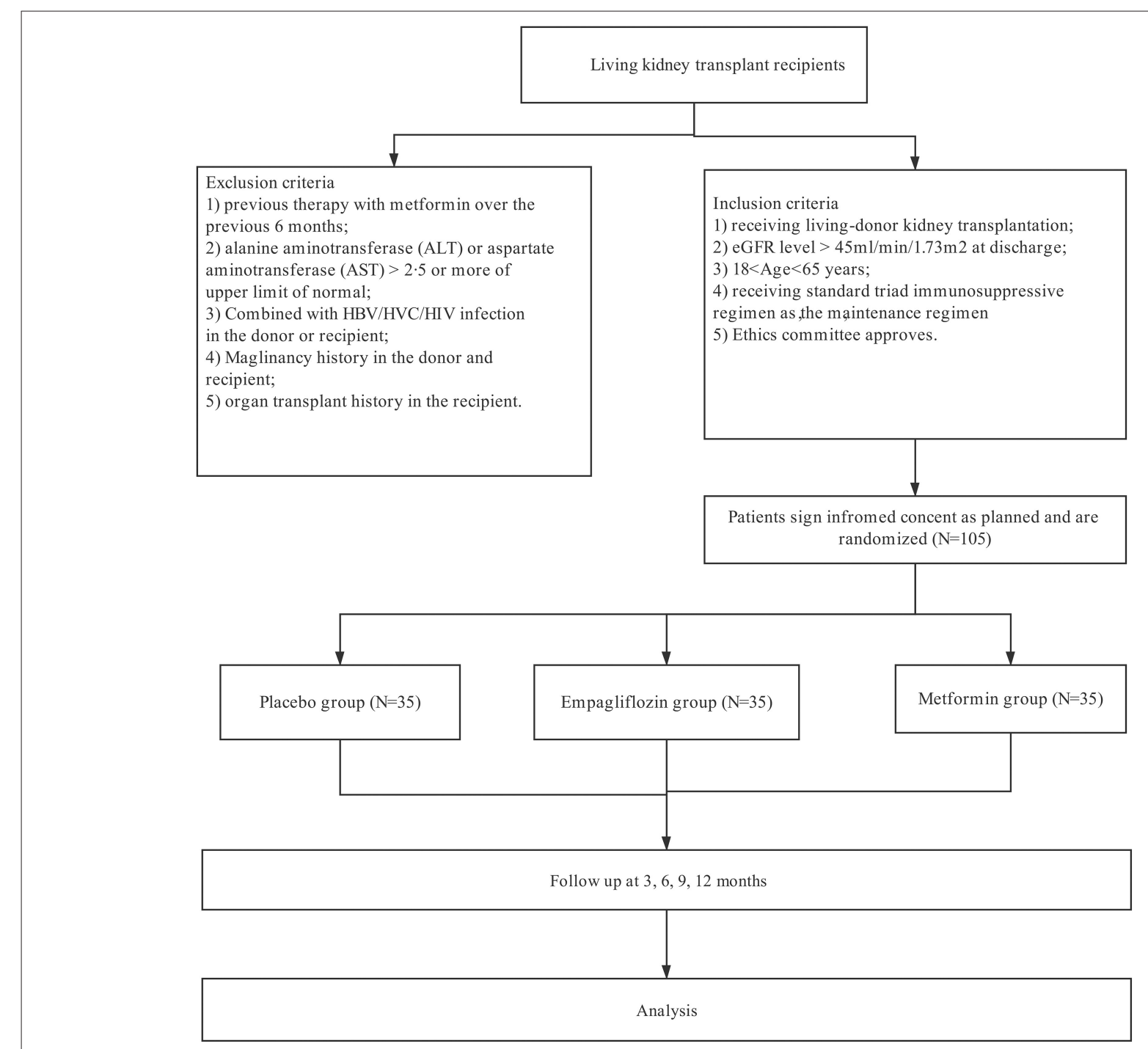

FIGURE 1 | Patients selection. 
TABLE 1 | Routine follow-up.

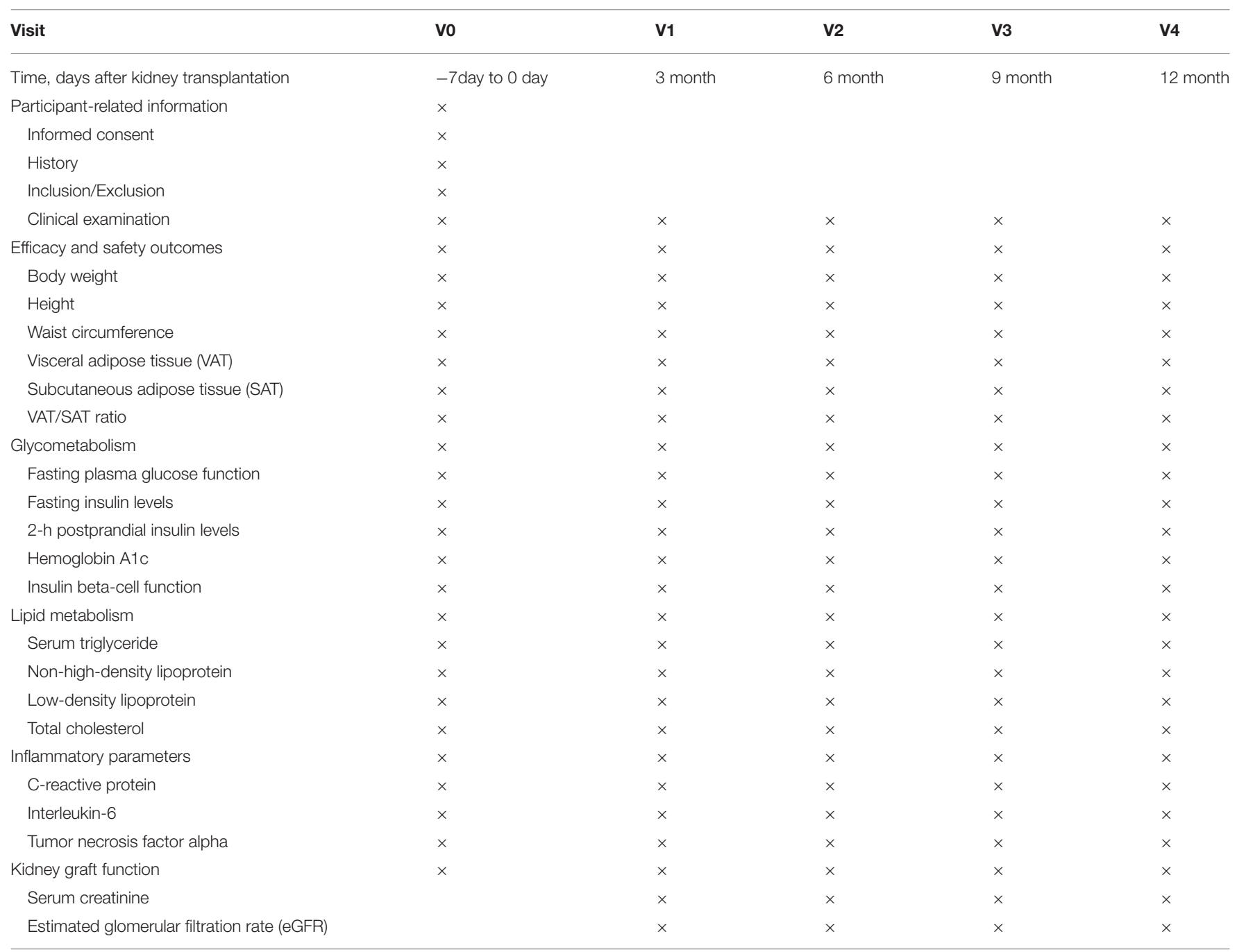

Differences between participants who complete and withdraw from the trial will be analyzed by using a Student $t$-test or the Mann-Whitney test for continuous variables (e.g., age) and the chi-squared test for categorical variables (e.g., sex). For clinical outcomes, analysis of covariance will be used to examine differences among three groups, adjusting for potential confounding factors and effect modifiers (e.g., baseline age and sex). Based on the literature, some patients have higher risk of post-transplantation diabetes mellitus (PTDM), including those with family history of diabetes; or polycystic kidney disease; or age $\geq 60$ years; or age $45-59$ years plus (i) triglycerides $\geq 200 \mathrm{mg} / \mathrm{dL}$; (ii) triglycerides $150-200 \mathrm{mg} / \mathrm{dL}$ and body mass index $>27 \mathrm{~kg} / \mathrm{m} 2$; (iii) triglycerides $150-200 \mathrm{mg} / \mathrm{dL}$ and high density lipoprotein (HDL) $<40 \mathrm{mg} / \mathrm{dL}(\mathrm{men}) /<40 \mathrm{mg} / \mathrm{dL}$ (women) Hence, subgroup analysis will be conducted based on the risk of PTDM, and was also conducted based on the components of the criteria for high-risk PTDM (26-28). Our statistical analysis will be performed by using R software, and the results will be considered significant at a $P$-value of $<0.05$.

\section{DISCUSSION}

This trial will provide more evidence on the safety and tolerability of metformin and SGLT2 agents in patients after renal transplantation. Most importantly, the study will also evaluate potential adjuvant therapies to ameliorating metabolic profiles in kidney recipients.

Successful developments in immunosuppression and clinical management make KT routines in adults with end-stage renal disease, but also unmask a greatly increased risk of premature metabolic disorders post-operatively. Generally accepted, long-term exposure to steroids can induce or worsen preexisting insulin resistance, increase hepatic gluconeogenesis, and stimulate appetite and weight gain (5). In addition, calcineurin inhibitors can inhibit sterol 26-hydroxylase, diminish hepatic bile acid synthesis, reduce export of cholesterol from the liver, and inhibit $\beta$-cell growth and function (6). Compared with the general population, high prevalence of metabolic disorders increases the risk of post-transplant CVD, which makes CVD a main post-transplant burden. In Chadban's 
study including 23210 first kidney-only transplant recipients from 1980 through 2018 from the Australia and New Zealand Dialysis and Transplant Registry, the authors assessed the trend of all-cause and cause-specific mortality at different periods post-transplant (29). Although morality declined over successive eras at all periods, CVD remained the most common cause of death despite of reduced account from 50\% in 1985-1989 to 30\% in 2015-2018. Further analysis showed that in the current era (2015-2018), the adjusted death rates due to CVD was $0.28,0.50$, 0.96 per 100 patient-years with a follow-up of $1-5,5-10$, and $>10$ years. However, less attention is focused on how to improve metabolic profiles in this population.

Previous studies demonstrated that therapeutics aimed at activating AMPK remain promising and beneficial for improving metabolic disorders in the context of obesity, diabetes, cancer, non-alcoholic fatty liver disease, cardiovascular diseases (7-10, 30-32). In Kulkarni's study, they studied participants $>70$ years $(N=14)$ in a randomized, double-blind, placebo-controlled, crossover trial in which they were treated with 6 weeks each of metformin and placebo (33). The authors observed that both metabolic and non-metabolic pathways were significantly influenced, including pyruvate metabolism, mitochondrial fatty acid oxidation, and collagen trimerization in adipose. Similar improvements of metabolic disorders were also reported in those patients diagnosed with cancer and treated by metformin in a well-designed clinical trial (34). Another recent clinical trial reported that metformin can improve metabolic profiles in Cushing's syndrome without diabetes and receiving systematic steroids $((\geq 20 \mathrm{mg} /$ day for $\geq 4$ weeks and remaining on $\geq 10$ $\mathrm{mg} /$ day for the subsequent 12 weeks, or its cumulative doseequivalent) (12). 53 patients were randomly assigned to receive either metformin $(n=26)$ or placebo $(n=27)$ for 12 weeks. Improvements in markers of carbohydrate, lipid, liver, and bone metabolism were observed in the metformin group compared with the placebo group. These studies demonstrated available benefits in metabolic profiles for patients without diabetes.

However, metformin was also associated with potential side effects, such as metformin-associated lactic acidosis (35). Metformin plasma concentrations are $\sim 2-4$ folds higher in patients with type 2 diabetes and moderate to severe renal impairment (eGFR of 30 to $<60 \mathrm{~mL} / \mathrm{min} / 1.73 \mathrm{~m} 2$ or $<30$ $\mathrm{mL} / \mathrm{min} / 1.73 \mathrm{~m} 2$, respectively) compared to healthy subjects (36). In a community-based cohort study, 75,413 patients with diabetes and time-dependent assessment of eGFR stage from January 2004 until January 2017 were included (37). The authors reported that there were 2335 hospitalizations with acidosis over a median follow-up of 5.7 years. Further analysis demonstrated that time-dependent metformin use was associated with incident acidosis only in patients with eGFR $<30 \mathrm{~mL} / \mathrm{min} / 1.73 \mathrm{~m} 2$ (HR: 2.07; 95\% CI: 1.33-3.22), but not in patients with eGFR 45$59 \mathrm{~mL} / \mathrm{min} / 1.73 \mathrm{~m} 2$ (HR: 1.16; 95\% CI: 0.95-1.41) and eGFR 30 to $44 \mathrm{~mL} / \mathrm{min} / 1.73 \mathrm{~m} 2$ (HR: 1.09 ; 95\% CI: $0.83-1.44$ ). This showed that metformin therapy may be safe in patients with eGFR $30-60 \mathrm{~mL} / \mathrm{min} / 1.73 \mathrm{~m} 2$. For kidney transplant recipients, evidence of metformin is insufficient. A recent small clinical trial demonstrated that metformin can be administered safely in transplant recipients with stable kidney graft function but impaired glucose tolerance $(N=19)$ (17). Hence, considering potential benefits of metformin, it is time to reassess the safety and value of metformin in kidney transplant patients.

Another first-line anti-diabetic drug, SGLT2 had definite cardiovascular and renal function protection in patients with or without diabetes reported by many large clinical trials (13-15). In addition to improving glycometabolism, large clinical trials showed that SGLT-2 inhibitors can improve metabolic parameters in patients with or without diabetes $(14,38)$. Also, this action mechanism is insulin-independent; as such it does not increase the risk of hypoglycemia, making it attractive for use in normoglycemic individuals (39). Davies et al. demonstrated that assessed the effect of canagliflozin on the components of metabolic syndrome of metabolic syndrome in patients with T2DM and metabolic syndrome (38). The authors indicated that canagliflozin can significantly reduce $\mathrm{HbAcl}$, fasting plasma glucose, body weight, body mass index, waist circumference, blood pressure, and triglycerides, and increased high-density lipoprotein cholesterol, and low-density lipoprotein cholesterol. Similar improvement of metabolic parameters was also seen in patients without diabetes. In the small clinical trial by Javed et al. the authors evaluated the effects of empagliflozin on metabolic parameters in polycystic ovary syndrome (16). Women with polycystic ovary syndrome were randomized to either empagliflozin $25 \mathrm{mg}(n=19)$ or metformin $1500 \mathrm{mg}$ $(n=20)$ daily for 12 weeks with the main outcomes as the changes in anthropometric and body composition, and metabolic parameters. Despite of no statistical difference in metabolic parameters between two groups, there was a significant improvement in anthropometric parameters and body composition, in patients receiving empagliflozin.

However, SGLT2 agents are also restricted in kidney transplant recipients due to their mechanism of elimination and action by concentrate glucose in the urine. This can increase risk of urinary tract infections $(40,41)$, especially in patients with immunosuppression. Two recent small clinical trials have evaluated the safety and efficacy of SGLT2 in treating diabetes after kidney transplantation $(18,19)$. In the trial by Halden, 22 renal transplant recipients with diabetes were randomized to receive $10 \mathrm{mg}$ empagliflozin with 20 receiving placebo as the control group once daily for 24 weeks. The authors shows that empagliflozin appeared safe and improved glycemic control in renal transplant recipients with NODAT compared with placebo. Also, a concomitant reduction in body weight was seen (443). In another preliminary prospective interventional trial including 14 kidney transplant recipients with NODAT and receiving insulin, the authors showed that empagliflozin can safely be used as addon therapy when monitored closely (454). The latest clinical trial even showed that dapagliflozin can improve kidney function and reduce the risk of end-stage kidney disease and cardiovascular death in patients with stage $4 \mathrm{CKD}$ and albuminuria (42). Hence, prospective studies are needed to explore the safety, tolerability and efficacy of metformin in improving metabolic profiles kidney transplant recipients with or without diabetes.

The limitation is that this study will explore the short-term impact of the intervention because the follow-up is set at 1 year. However, the development of metabolic diseases is a long and gradual process and can be influenced by other factors. 
In summary, the AMPKT study will be the first dedicated clinical trial to explore the potential benefits and risks of metformin and SGLT2 inhibitors in kidney transplant recipients both with and without diabetes.

\section{ETHICS STATEMENT}

The studies involving human participants were reviewed and approved by the Ethics Committee of West China Hospital. Written informed consent was not provided because this is a protocol for a Clinical Trial.

\section{AUTHOR CONTRIBUTIONS}

SY, XW, TL, and TS were the initiators of this study. SY and MM wrote this protocol. SY, XW, TS, ZH, YF, and TL tool part in

\section{REFERENCES}

1. Hart A, Lentine KL, Smith JM, Miller JM, Skeans MA, Prentice M, et al. OPTN/SRTR (2019). Annual data report: kidney. Am J Transplant. (2021) 21(Suppl. 2):21-137. doi: 10.1111/ajt.16502

2. Stoumpos S, Jardine AG, Mark PB. Cardiovascular morbidity and mortality after kidney transplantation. Transpl Int. (2015) 28:10-21. doi: 10.1111/tri.12413

3. Piotti G, Gandolfini I, Palmisano A, Maggiore U. Metabolic risk profile in kidney transplant candidates and recipients. Nephrol Dial Transplant. (2019) 34:388-400. doi: 10.1093/ndt/gfy151

4. Cohen E, Korah M, Callender G, Belfort de Aguiar R, Haakinson D. Metabolic disorders with kidney transplant. Clin J Am Soc Nephrol. (2020) 15:73242. doi: 10.2215/CJN.09310819

5. Pivonello R, Isidori AM, De Martino MC, Newell-Price J, Biller BM, Colao A. Complications of Cushing's syndrome: state of the art. Lancet Diabetes Endocrinol. (2016) 4:611-29. doi: 10.1016/S2213-8587(16)00086-3

6. Marchetti P, Navalesi R. The metabolic effects of cyclosporin and tacrolimus. $J$ Endocrinol Invest. (2000) 23:482-90. doi: 10.1007/BF03343761

7. Zhang BB, Zhou G, Li C, AMPK. an emerging drug target for diabetes and the metabolic syndrome. Cell Metab. (2009) 9:407-16. doi: 10.1016/j.cmet.2009.03.012

8. Ruderman NB, Carling D, Prentki M, Cacicedo JM. AMPK insulin resistance, and the metabolic syndrome. J Clin Invest. (2013) 123:276472. doi: $10.1172 /$ JCI67227

9. Carling D. AMPK signalling in health and disease. Curr Opin Cell Biol. (2017) 45:31-7. doi: 10.1016/j.ceb.2017.01.005

10. Day EA, Ford RJ, Steinberg GR. AMPK as a therapeutic target for treating metabolic diseases. Trends Endocrinol Metab. (2017) 28:54560. doi: 10.1016/j.tem.2017.05.004

11. Zhou G, Myers R, Li Y, Chen Y, Shen X, Fenyk-Melody J, et al. Role of AMPactivated protein kinase in mechanism of metformin action. J Clin Invest. (2001) 108:1167-74. doi: 10.1172/JCI13505

12. Pernicova I, Kelly S, Ajodha S, Sahdev A, Bestwick JP, Gabrovska P, et al. Metformin to reduce metabolic complications and inflammation in patients on systemic glucocorticoid therapy: a randomised, double-blind, placebocontrolled, proof-of-concept, phase 2 trial. Lancet Diabetes Endocrinol. (2020) 8:278-91. doi: 10.1016/S2213-8587(20)30021-8

13. Zinman B, Wanner C, Lachin JM, et al. Empagliflozin, cardiovascular outcomes, and mortality in type 2 diabetes. N Engl J Med. (2015) 373:211728. doi: 10.1056/NEJMoa1504720

14. Cahn A, Raz I, Leiter LA, Mosenzon O, Murphy SA, Goodrich EL, et al. Cardiovascular, renal, and metabolic outcomes of dapagliflozin versus placebo in a primary cardiovascular prevention cohort: analyses from DECLARETIMI 58. Diabetes Care. (2021) 44:1159-67. doi: 10.2337/dc20-2492 the study planning. SY and XW wrote the final manuscript. All authors have read and approved the final manuscript.

\section{FUNDING}

This work was supported by grants from the National Natural Science Foundation of China [Grant Number 81870513]; Sichuan Science and Technology Program [Grant Number 2019YJ0133]; Chengdu Science and Technology Program [Grant Number 2019-YF05-00084-SN]; and 1.3.5 Project for Disciplines of Excellence-Clinical Research Incubation Project, West China Hospital, Sichuan University [Grant Numbers 2018HXFH049, ZYJC18004, ZY2016104, and 2021HXFH007]. The funders had no role in study design, data collection or analysis, preparation of the manuscript, or the decision to publish.

15. Perakakis N, Chrysafi P, Feigh M, Veidal SS, Mantzoros CS. Empagliflozin improves metabolic and hepatic outcomes in a non-diabetic obese biopsy-proven mouse model of advanced NASH. Int J Mol Sci. (2021) 22:6332. doi: 10.3390/ijms22126332

16. Javed Z, Papageorgiou M, Deshmukh H, Rigby AS, Qamar U, Abbas J, et al. Effects of empagliflozin on metabolic parameters in polycystic ovary syndrome: a randomized controlled study. Clin Endocrinol (Oxf). (2019) 90:805-13. doi: 10.1111/cen.13968

17. Alnasrallah B, Goh TL, Chan LW, Manley P, Pilmore H. Transplantation and diabetes (Transdiab): a pilot randomised controlled trial of metformin in impaired glucose tolerance after kidney transplantation. BMC Nephrol. (2019) 20:147. doi: 10.1186/s12882-019-1321-2

18. Halden TAS, Kvitne KE, Midtvedt K, Rajakumar L, Robertsen I, Brox J, et al. Efficacy and safety of empagliflozin in renal transplant recipients with posttransplant diabetes mellitus. Diabetes Care. (2019) 42:106774. doi: $10.2337 / \mathrm{dc} 19-0093$

19. Schwaiger E, Burghart L, Signorini L, Ristl R, Kopecky C, Tura A, et al. Empagliflozin in posttransplantation diabetes mellitus: a prospective, interventional pilot study on glucose metabolism, fluid volume, and patient safety. Am J Transplant. (2019) 19:907-19. doi: 10.1111/ajt.15223

20. Patorno E, Pawar A, Franklin JM, Najafzadeh $M$, Déruaz-Luyet A, Brodovicz KG, et al. Empagliflozin and the risk of heart failure hospitalization in routine clinical care. Circulation. (2019) 139:2822-30. doi: 10.1161/CIRCULATIONAHA.118.039177

21. The Chinese Society of Organ Transplantation. Chinese management guideline of post-transplantation diabetes mellitus. Organ Transpl. (2019) 10:1-10.

22. Yin $S$, Wang $X$, Huang Z, Fan $Y$, Song T, Lin T. Tacrolimus variability score outperforms coefficient of variation in predicting clinical outcomes of living kidney transplantation. Br J Clin Pharmacol. (2021). doi: 10.1111/bcp.14876

23. Rockall AG, Sohaib SA, Evans D, Kaltsas G, Isidori AM, Monson JP, et al. Computed tomography assessment of fat distribution in male and female patients with Cushing's syndrome. Eur J Endocrinol. (2003) 149:56167. doi: 10.1530/eje.0.1490561

24. Kaess BM, Pedley A, Massaro JM, Murabito J, Hoffmann U, Fox CS. The ratio of visceral to subcutaneous fat, a metric of body fat distribution, is a unique correlate of cardiometabolic risk. Diabetologia. (2012) 55:262230. doi: 10.1007/s00125-012-2639-5

25. Mitsui Y, Sadahira T, Araki M, et al. Clinical impact of abdominal fat distribution measured by 3-D computed tomography volumetry on post-transplant renal function in recipients after living kidney transplantation: a retrospective study. Clin Exp Nephrol. (2019) 23:415-24. doi: 10.1007/s10157-018-1643-6

26. Cosio FG, Pesavento TE, Kim S, Osei K, Henry M, Ferguson RM: patient survival after renal transplantation: IV. Impact of post-transplant 
diabetes. Kidney Int. (2002) 62:1440-16. doi: 10.1111/j.1523-1755.2002.kid 582.x

27. Romagnoli J, Citterio F, Violi P, Cadeddu F, Nanni G, Castagneto M: Post-transplant diabetes mellitus: a case-control analysis of the risk factors. Transpl Int. (2005) 18:309-12. doi: 10.1111/j.1432-2277.2004.00 043.x

28. Shah T, Kasravi A, Huang E, Hayashi R, Young B, Cho YW, et al. Risk factors for development of new-onset diabetes mellitus after kidney transplantation. Transplantation. (2006) 82:1673-6. doi: 10.1097/01.tp.0000250756.66 348.9a

29. Ying T, Shi B, Kelly PJ, Pilmore H, Clayton PA, Chadban SJ. Death after kidney transplantation: an analysis by era and time post-transplant. J Am Soc Nephrol. (2020) 31:2887-99. doi: 10.1681/ASN.2020050566

30. Duca FA, Côté CD, Rasmussen BA, Zadeh-Tahmasebi M, Rutter GA, Filippi BM, et al. Metformin activates a duodenal Ampk-dependent pathway to lower hepatic glucose production in rats. Nat Med. (2015) 21:50611. doi: $10.1038 / \mathrm{nm} .3787$

31. Cool B, Zinker B, Chiou W, Kifle L, Cao N, Perham M, et al. Identification and characterization of a small molecule AMPK activator that treats key components of type 2 diabetes and the metabolic syndrome. Cell Metab. (2006) 3:403-16. doi: 10.1016/j.cmet.2006.05.005

32. Foretz M, Hébrard S, Leclerc J, Zarrinpashneh E, Soty M, Mithieux G, et al. Metformin inhibits hepatic gluconeogenesis in mice independently of the LKB1/AMPK pathway via a decrease in hepatic energy state. J Clin Invest. (2010) 120:2355-69. doi: 10.1172/JCI40671

33. Kulkarni AS, Brutsaert EF, Anghel V, Zhang K, Bloomgarden N, Pollak M, et al. Metformin regulates metabolic and nonmetabolic pathways in skeletal muscle and subcutaneous adipose tissues of older adults. Aging Cell. (2018) 17:e12723. doi: 10.1111/acel.12723

34. Goodwin PJ, Dowling RJO, Ennis M, Chen BE, Parulekar WR, Shepherd LE, et al. Effect of metformin versus placebo on metabolic factors in the MA.32 randomized breast cancer trial. NPJ Breast Cancer. (2021) 7:74. doi: 10.1038/s41523-021-00275-z

35. Flory JH, Hennessy S, Bailey CJ, Inzucchi SE. Reports of lactic acidosis attributed to metformin, 2015-2018. Diabetes Care. (2020) 43:244-6. doi: 10.2337/dc19-0923

36. Frid A, Sterner GN, Löndahl M, Wiklander C, Cato A, Vinge E, et al. Novel assay of metformin levels in patients with type 2 diabetes and varying levels of renal function: clinical recommendations. Diabetes Care. (2010) 33:12913. doi: $10.2337 / \mathrm{dc} 09-1284$
37. Lazarus B, Wu A, Shin JI, Sang Y, Alexander GC, Secora A, et al. Association of metformin use with risk of lactic acidosis across the range of kidney function: a community-based cohort study. JAMA Intern Med. (2018) 178:903-10. doi: 10.1001/jamainternmed.2018.0292

38. Op den. Kamp YJM, de Ligt M, Dautzenberg B, Kornips E, Esterline R, Hesselink MKC, et al. Effects of the SGLT2 inhibitor dapagliflozin on energy metabolism in patients with type 2 diabetes: a randomized, double-blind crossover trial. Diabetes Care. (2021) 15:dc202887. doi: 10.2337/dc20-2887

39. Scheen AJ. Pharmacokinetic and pharmacodynamic profile of empagliflozin, a sodium glucose co-transporter 2 inhibitor. Clin Pharmacokinet. (2014) 53:213-25. doi: 10.1007/s40262-013-0126-x

40. Hecking $M$, Jenssen T. Considerations for SGLT2 inhibitor use in post-transplantation diabetes. Nat Rev Nephrol. (2019) 15:525-6. doi: 10.1038/s41581-019-0173-0

41. Chowdhury TA, Wahba M, Mallik R, et al. Association of British Clinical Diabetologists and Renal Association guidelines on the detection and management of diabetes post solid organ transplantation. Diabet Med. (2021) 38:e14523. doi: 10.1111/dme.14523

42. Chertow G, Vart P, Jongs N, Toto R, Gorriz JL, Hou FF, et al. Effects of dapagliflozin in stage 4 chronic kidney disease. J Am Soc Nephrol. (2021) 32:2352-61. doi: 10.1681/ASN.2021020167

Conflict of Interest: The authors declare that the research was conducted in the absence of any commercial or financial relationships that could be construed as a potential conflict of interest.

Publisher's Note: All claims expressed in this article are solely those of the authors and do not necessarily represent those of their affiliated organizations, or those of the publisher, the editors and the reviewers. Any product that may be evaluated in this article, or claim that may be made by its manufacturer, is not guaranteed or endorsed by the publisher.

Copyright (C) 2021 Yin, Ma, Huang, Fan, Wang, Song and Lin. This is an open-access article distributed under the terms of the Creative Commons Attribution License (CC $B Y)$. The use, distribution or reproduction in other forums is permitted, provided the original author(s) and the copyright owner(s) are credited and that the original publication in this journal is cited, in accordance with accepted academic practice. No use, distribution or reproduction is permitted which does not comply with these terms. 